\title{
Produção de aguardente de ciriguela (Spondias purpurea L.) utilizando diferentes cepas de Saccharomyces cerevisiae
}

\author{
Production of ciriguela (Spondias purpurea L.) spirits using \\ different Saccharomyces cerevisiae strains.
}

'Universidade Federal da Grande Dourados - UFGD, R. João Rosa Góes, 1761, CEP 79825-070, Vila Progresso, Dourados, MS, Brasil

* autor correspondente $\nabla$ daniellevilela@ufgd.edu.br

Hevila Thainara dos Santos ${ }^{1}$, Wenia Furtado Lima', Gustavo Graciano Fonseca' Danielle Marques Vilela ${ }^{1 *}$

RESUMO: A ciriguela (Spondias purpurea L.) é uma fruta típica do cerrado, com características essenciais para a fermentação alcoólica por $S$. cerevisiae $\left(21,25^{\circ}\right.$ Brix $)$. O objetivo deste trabalho foi elaborar aguardentes com polpa dos frutos ciriguela utilizando 4 cepas de $S$. cerevisiae (LNFCA11®; LEVSIL16 e LEVSIL5; LEVFRC29). A produção do mosto de ciriguela foi realizada em duplicata em elernmeyers, contendo $250 \mathrm{~mL}$ de polpa de ciriguela a $16^{\circ}$ Brix. Os cultivos foram realizados em sistema de batelada simples para cada cepa. Os vinhos obtidos foram submetidos ao processo de destilação fracionada em alambique de cobre e logo após feita análise em HPLC. Das bebidas de ciriguela produzidas, as cepas (LEVFRC29) e (LNFCA11®) resultaram em aguardentes com graduações alcoólicas (GL) 38,44 e $45,96\left({ }^{\circ} \mathrm{GL}\right)$, respectivamente. A bebida produzida pelo isolado LEVSIL16 $(208 \mathrm{mg} / 100 \mathrm{~mL})$ teve uma quantidade de ácido acético maior que o permitido na legislação $(100 \mathrm{mg} / 100 \mathrm{~mL})$. Para os valores de glicerol, LEVSIL15 resultou em uma bebida com maior valor de glicerol $(0,09 \mathrm{~g} / \mathrm{L})$. As cepas que obtiveram uma melhor produtividade de etanol foram LNFCA11 ${ }^{\circledR}\left(4,18 \mathrm{~g} / \mathrm{L} /{ }^{\circ} \mathrm{Brix}\right) \mathrm{e}$ LEVFRC29 (3,49 g/L/ ${ }^{\circ}$ Brix). O presente estudo foi o primeiro para viabilizar o processo de produção de aguardente de ciriguela. $\mathrm{O}$ isolado comercial (LNFCA11®) foi eficiente na fermentação alcoólica da polpa de ciriguela e para a produção de aguardente. A cepa LEVFRC29 mostrou-se promissora, pois resultou em uma aguardente de ciriguela com características semelhantes à comercial. Concluindo que a cepa comercial LNFCA11 ${ }^{\circledR}$ e LEVFRC29 obtiveram os melhores resultados para produção da aguardente.

PALAVRAS-CHAVE: Fermentação, fruta, isolados.
ABSTRACT: Ciriguela (Spondias purpurea L.) is a typical cerrado fruit with essential characteristics for alcoholic fermentation by Sacharomyces cerevisiae (21.25 ${ }^{\circ}$ Brix). This work aimed to prepare spirits with the pulp of ciriguela fruits using 4 strains of $S$. cerevisiae (LNFCA11 ${ }^{\circledR}$; LEVSIL16; LEVSIL5; LEVFRC29). The production of the ciriguela must was carried out in duplicate in Erlenmeyer flasks containing $250 \mathrm{~mL}$ of ciriguela pulp at $16^{\circ}$ Brix. Cultivations were carried out in a simple batch system for each strain. The ciriguela wines obtained were subjected to the fractional distillation process in a copper still before HPLC analysis. The strains LEVFRC29 and LNFCA11 ${ }^{\circledR}$ resulted in spirits with alcoholic grades of 38.44 and 45.96 (GL), respectively. The drink produced from the LEVSIL 16 strain $(208 \mathrm{mg} / 100 \mathrm{~mL})$ had an amount of acetic acid greater than that allowed by the Brazilian legislation $(100 \mathrm{mg} / 100 \mathrm{~mL}$ ) while the LEVSIL 15 strain resulted in a drink with a higher glycerol value $(0.09 \mathrm{~g} / \mathrm{L})$. The strains with higher ethanol productivities were $L N F C A 11 \AA\left(4.18 \mathrm{~g} / \mathrm{L} /{ }^{\circ} \mathrm{Brix}\right)$ and LEVFRC29 (3.49 $\left.\mathrm{g} / \mathrm{L}{ }^{\circ} \mathrm{Brix}\right)$. It underlines that the commercial isolate (LNFCA11 $\AA$ ) was more efficient in the alcoholic fermentation of the ciriguela pulp and that the LEVFRC29 strain resulted in a ciriguela spirit with characteristics similar to the commercial ones. It was concluded that the LNFCA11® and LEVFRC29 strains presented the best results for the production of spirits from ciriguela pulp.

KEYWORDS: Fermentation, fruit, isolated. 


\section{Introdução}

Segundo o Ministério de Agricultura, Pecuária e Abastecimento (MAPA), é definida como cachaça "a aguardente de cana produzida no Brasil, com graduação alcoólica (GL) de 38 a 48\%, a $20^{\circ} \mathrm{C}$, obtida pela destilação do mosto fermentado do caldo de cana-de-açúcar com características sensoriais peculiares, podendo ser adicionada de açúcares até seis gramas por litro ( $\leq$ $6 \mathrm{~g} / \mathrm{L}$ de açúcar). Já aguardente (derivada de outra matéria-prima que não a cana) é a bebida com graduação alcoólica (GL) de 38 a $54 \%$, a $20^{\circ} \mathrm{C}$, obtida do rebaixamento do teor alcoólico do destilado alcoólico simples ou pela destilação do mosto fermentado" (BRASIL, 2019).

$\mathrm{Na}$ legislação, a aguardente obtida a partir de frutas pode ser denominada aguardente de frutas ou brandy de frutas, com GL de 38 a $54 \%$, podendo ser simples ou envelhecida. As bebidas que não passam por envelhecimento podem ser denominadas simplesmente aguardente, e seguida do nome da fruta da qual é obtida (LIMA, 2001).

No Brasil, a cachaça é a segunda bebida alcoólica mais consumida e a primeira em relação às bebidas destiladas (ALCARDE; MONTEIRO; BELLUCO, 2012). Apesar de o fermentado de cana ser bastante popular, a produção de aguardente a partir de frutas vem sendo uma opção para os produtores, pois diminui as perdas pós-colheita e agrega valor à fruta, além de serem novas composições de sabores para os consumidores (VIEIRA, 2012).

A ciriguela (Spondias purpurea L.) é uma fruta da família Anacardiaceae, comum do cerrado, bastante comercializada devido ao sabor e à cor atrativa, porém é muito perecível, por isso procuram-se alternativas para que a fruta não seja desperdiçada, como, por exemplo, a produção das bebidas fermentadas (SILVA; FIGUEIREDO; LIMA, 2016). A ciriguela, quando madura, apresenta características físico-químicas de $21,25 \%$ de sólidos solúveis totais ( $\left.{ }^{\circ} \mathrm{Brix}\right)$, açúcares solúveis totais de $18,68 \%$, açúcares redutores de $0,70 \%$ e amido $1 \%$ (MARTINS; MELO, 2012). Bons parâmetros, se comparados ao caldo de cana-de-açúcar madura, onde os açúcares redutores são menores que $1 \%$ e a sacarose é em torno de $14 \%$ ou mais (aproximadamente $18^{\circ}$ Brix) (LIMA; BASSO; AMORIM, 2001).

Para a obtenção da aguardente, a matéria-prima, seja cana-de-açúcar, frutas ou cereais, precisa passar pelo processo de transformação dos açúcares presentes em sua composição em álcool etílico e $\mathrm{CO}_{2}$, e para que isso aconteça, é necessário a ação de leveduras, como Saccharomyces cerevisiae (MENDONÇA et al., 2014).

Bebidas fermentadas derivadas de frutos são uma opção para obtenção de produtos com maior vida útil, além disso, agregam mais valor às bebidas (MUNIZ et al., 2002). A ciriguela, por exemplo, possui vitamina $\mathrm{C}$ e provitamina $\mathrm{A}$, que podem ser agregadas à bebida e trazer benefícios à saúde, já que vitaminas são estudadas como forma de prevenção de algumas doenças (LIMA; MELEIRO, 2012). Nesse sentido, o objetivo deste trabalho foi elaborar aguardentes de ciriguela (Spondias purpurea L.) utilizando 4 cepas de $S$. cerevisiae LNFCA11 ${ }^{\circledR}$ - Cepa comercial; LEVSIL16 e LEVSIL15 - isolados obtidos da silagem de capim Piatã; LEVFRC29 - isolado proveniente de frutos do cerrado, sendo as últimas três cepas pertencentes à coleção microrganismos do grupo de pesquisa.

\section{Materiais e Métodos}

O experimento foi desenvolvido no Laboratório de Bioquímica da Faculdade de Ciências Biológicas e Ambientais, Universidade Federal da Grande Dourados - UFGD Dourados, MS, Brasil. Na Figura 1 demonstra-se o fluxograma das etapas feitas durante todo o processo para obtenção de aguardente de ciriguela.

\section{Obtenção da polpa de ciriguela}

Parte dos frutos de ciriguela foi adquirida em feiras populares de Dourados - MS, e outra parte foi coletada na zona rural da cidade de Rio Brilhante - MS no assentamento Taubinha. Após serem lavadas com água corrente e higienizadas com solução de hipoclorito de sódio $1 \%(\mathrm{v} / \mathrm{v})$ por cerca de 2 minutos, as frutas foram despolpadas manualmente com o auxílio de peneiras de alumínio. A polpa obtida a $16^{\circ}$ Brix foi acondicionada em sacos plásticos e posteriormente armazenada em freezer a $-15^{\circ} \mathrm{C}$.

\section{Produção do inóculo}

Foram testados 4 isolados de $S$. cerevisiae (LNFCA11 ${ }^{\circledR}$ - Cepa comercial; LEVSIL16 e LEVSIL5 - isolados obtidos da silagem de capim Piatã; LEVFRC29 - isolado proveniente de frutos do cerrado). As cepas foram reativadas em caldo YPD ( $1 \%$ de extrato de levedura, $2 \%$ de glicose e $2 \%$ de peptona $\mathrm{m} / \mathrm{v}$ ). Para a multiplicação do inóculo, uma alçada da cultura estoque foi

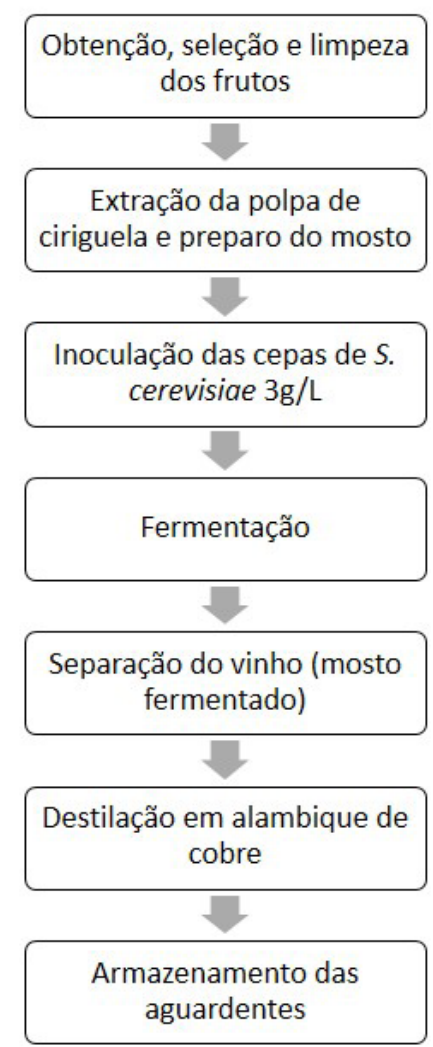

Figura 1. Fluxograma do processo de produção de aguardente de ciriguela (Spondias purpurea L.). 
transferida para tubos de ensaio contendo $5 \mathrm{~mL}$ de meio YPD e incubadas a $30{ }^{\circ} \mathrm{C}$ por 24 horas. Dois $\mathrm{mL}$ da suspensão de células foram transferidos, com auxílio de uma micropipeta, para tubos de ensaio contendo $10 \mathrm{~mL}$ de YPD e incubados em BOD a $30^{\circ} \mathrm{C}$ por 24 horas. Cinco $\mathrm{mL}$ da suspensão de células foram transferidos para erlenmeyers contendo $200 \mathrm{~mL}$ de YPD e incubados a $30{ }^{\circ} \mathrm{C}$, sob agitação $(100 \mathrm{rpm})$ por 24 horas, a fim de que a população do inóculo atingisse a contagem de $10^{6} \mathrm{UFC} / \mathrm{mL}$ na Câmara de Neubauer.

\section{Fermentação de polpa de ciriguela}

A produção do vinho de ciriguela foi realizada em duplicata em elernmeyers de $500 \mathrm{~mL}$, contendo $250 \mathrm{~mL}$ de polpa de ciriguela a $16{ }^{\circ}$ Brix para cada isolado, com inóculo de $3 \mathrm{~g} / \mathrm{L}$ de massa celular. Os cultivos foram realizados em sistema de batelada simples a $30^{\circ} \mathrm{C}$, sendo que a cada 12 horas de cultivo foram realizadas leituras de sólidos solúveis totais ( $\left.{ }^{\circ} \mathrm{Brix}\right)$, com o auxílio de um refratômetro portátil.

\section{Destilação}

Após a etapa de fermentação, os vinhos obtidos foram separados das leveduras e submetidos ao processo de destilação fracionada.

Em alambique de cobre equipado com condensador e aquecedor a gás e com capacidade de trabalho de $20 \mathrm{~L}$, a temperatura do mosto de ciriguela fermentado foi mantida entre 80 e $90{ }^{\circ} \mathrm{C}$. $\mathrm{O}$ destilado foi separado em três frações. A primeira fração (denominada de cabeça) foi coletada separadamente e padronizada para um volume correspondente a aproximadamente $10 \%$ do volume total da aguardente. A fração intermediária (denominada de coração) foi então coletada até a porção correspondente a $70 \%$ do volume total de aguardente. A última fração (denominada de cauda), correspondente a $20 \%$ do volume de aguardente produzida, também foi coletada e armazenada em frascos de vidro âmbar e mantida à temperatura ambiente variando de $18^{\circ}$ a $28^{\circ} \mathrm{C}$. A bebida final, a fração coração foi utilizada para a realização das análises físico-químicas.

\section{Análise HPLC}

Álcoois superiores (glicerol, propanol e etanol) ácidos orgânicos (ácidos acético, cítrico, succínico e lático) foram quantificados por cromatografia líquida de alta eficiência (HPLC), utilizando um cromatógrafo Shimadzu, modelo LC-10Ai (ShimadzuCorp.,
Japão), equipado com um sistema de detecção duplo que consiste em um detector de UV (SPD-10Ai) e um detector de índice de refração (RID-10Ai). Uma coluna de exclusão de íons Shimadzu (Shim-pack SCR-101H, 7,9 mm $\times 30 \mathrm{~cm}$ ) operada a uma temperatura de $55^{\circ} \mathrm{C}$ foi usada para alcançar a separação cromatográfica. A quantificação de álcoois e ácidos foi realizada por meio de curvas de calibração obtidas a partir de compostos padrão. Todas as amostras foram analisadas em duplicata.

\section{Análises estatîsticas}

As análises estatísticas (teste de Tukey a 5\%) foram conduzidas no programa Minitab.

\section{Resultados e Discussão}

O processo fermentativo da polpa de ciriguela iniciou com inóculo de $10^{3}$ células $/ \mathrm{mL}$, atingindo-se picos de contagem populacional de $10^{6}$ células $/ \mathrm{mL}$ com $48 \mathrm{~h}$. O consumo total de açúcares se deu em média com $72 \mathrm{~h}$ de cultivo, momento em que o caldo fermentado foi destilado em alambique de cobre.

De acordo com a tabela, podemos observar que a maior média de glicerol nas aguardentes foi produzida pela cepa $S$. cerevisiae LEVSIL15 (0,09 g/L) (Tabela 1), que se diferencia estatisticamente das demais cepas, a menor média foi para cepa $S$. cerevisiae LEVSIL16 (0,01 g/L) (Tabela 1), sendo essa estatisticamente igual aos isolados $S$. cerevisiae LNFCA $11 @(0,02 \mathrm{~g} / \mathrm{L})$, S. cerevisiae LEVFRC29 (0,02 g/L) (Tabela 1).

Quanto aos teores de ácido acético nas bebidas, aquela fermentada pela S. cerevisiae LEVSIL16 (208 mg/100 mL) (Tabela 1) apresentou a maior quantidade de ácido acético, diferenciando estatisticamente das demais. Já S. cerevisiae LNFCA11 ${ }^{\circledR}(9,95 \mathrm{mg} / 100 \mathrm{~mL})$ (Tabela 1) obteve o menor teor de ácido acético, porém não apresenta diferença estatística entre as cepas $S$. cerevisiae LEVFRC29 $(37,20 \mathrm{mg} / 100 \mathrm{~mL})$ e S. cerevisiae LEVSIL15 (28,80 mg/100 mL) (Tabela 1).

Em relação ao grau alcoólico das aguardentes, o maior GL foi produzido pela cepa comercial (S. cerevisiae LNFCA11 ${ }^{\circledR}$ 45,96 g/L) (Tabela 1), que é diferente estatisticamente das demais cepas. A cepa S. cerevisiae LEVSIL16 obteve o menor GL (14,56 g/L) (Tabela 1), diferenciando estatisticamente dos demais isolados.

Das aguardentes de ciriguela produzidas, aquelas fermentadas pelas cepas S. cerevisiae LEVFRC29 e S. cerevisiae $\operatorname{LNFCA11\circledR ~(38,~}$

Tabela 1. Análise por HPLC das aguardentes de ciriguelas fermentadas por 4 cepas de S. cerevisiae.

\begin{tabular}{|c|c|c|c|c|c|c|}
\hline \multirow{2}{*}{ Isolados } & \multirow{2}{*}{ Glicerol (g/L) } & \multirow{2}{*}{$\begin{array}{l}\text { Ácido Acético } \\
\text { (mg/100 mL) }\end{array}$} & \multirow{2}{*}{ GL (g/L) } & \multicolumn{2}{|c|}{${ }^{\circ}$ Brix } & \multirow{2}{*}{$\begin{array}{c}\text { Produtividade } \\
\text { (g/L/L }{ }^{\circ} \text { Brix) }\end{array}$} \\
\hline & & & & Inicial & Final & \\
\hline $\begin{array}{l}\text { S. cerevisiae } \\
\text { LEVSIL15 }\end{array}$ & $0,09 \mathrm{~A}$ & 28,80 B & $32,22 \mathrm{C}$ & 16 & 6 & $3,22 \mathrm{C}$ \\
\hline $\begin{array}{l}\text { S. cerevisiae } \\
\text { LEVFRC29 }\end{array}$ & 0,02 B & 37,20 B & 38,44 B & 16 & 5 & 3,49 B \\
\hline $\begin{array}{l}\text { S. cerevisiae } \\
\text { LNFCA11 }\end{array}$ & 0,02 B & 9,95 B & 45,96 A & 16 & 5 & $4,18 \mathrm{~A}$ \\
\hline $\begin{array}{l}\text { S. cerevisiae } \\
\text { LEVSIL16 }\end{array}$ & 0,01 B & $208 \mathrm{~A}$ & $14,56 \mathrm{D}$ & 16 & 9 & 2,08 D \\
\hline
\end{tabular}

Médias que não compartilham uma letra são estatisticamente diferentes. 
44 e 45,96 g/L, respectivamente) (Tabela 1) se encontram dentro dos padrões de graduação alcóolica estabelecidos pelo MAPA para aguardentes (38 a $54 \mathrm{~g} / \mathrm{L}$ ou \%). Já as aguardentes produzidas pelas cepas $S$. cerevisiae LEVSIL15 e S. cerevisiae LEVSIL16 apresentaram graduações alcoólicas abaixo do padrão (32,22 e 14,56 g/L, respectivamente) (Tabela 1), não sendo, portanto, classificadas como aguardentes.

Comparando os resultados da nossa aguardente com trabalhos feitos com aguardente de cana-de-açúcar (cachaça), podemos observar que as aguardentes produzidas neste trabalho tiveram bons resultados, como, por exemplo, em um estudo em que três cepas de $S$. cerevisiae foram utilizadas para a produção de cachaça, mostrou que duas das três cepas tiveram bebidas com variação nos teores alcoólicos de 40 a $45 \mathrm{GL}$, e que uma delas apresentou um valor um pouco maior $48 \mathrm{GL}$. Das cepas obtidas nesse trabalho, apenas a cepa comercial conseguiu atingir uma graduação alcoólica parecida (CAMPOS et al., 2010).

Em um trabalho realizado para caracterizar aguardentes artesanais de cana-de-açúcar, mostrou que, para a produção de cachaça, a acidez volátil expressa em ácido acético foi em média de 45,6 mg/100 mL (MENDES FILHO et al., 2016), ou seja, maior do que para a produção de aguardente de ciriguela, exceto pelo isolado $S$. cerevisiae LEVSIL16, que produziu uma quantidade de ácido acético maior que o permitido para aguardente de frutas, que em legislação consta que seria $100 \mathrm{mg} / 100 \mathrm{~mL}$ (BRASIL, 2011). Uma quantidade muita alta de ácido acético na aguardente pode ser causada por contaminação de bactérias ácido acéticas ou devido à presença de oxigênio, e sua presença pode influenciar negativamente no aroma e sabor da bebida (MARINHO; RODRIGUES; SIQUEIRA, 2009; PEREIRA et al., 2003).

Para o glicerol, em comparação com os resultados obtidos em um trabalho em que foi feito um experimento para avaliar a quantidade de glicerol em 52 cachaças envelhecidas e descansadas de vários estados, a média de glicerol para envelhecidas foi de $17 \mathrm{mg} / \mathrm{L}$, e para descansadas foi de $1,72 \mathrm{mg} / \mathrm{L}$. O presente trabalho apresenta valores mais altos de glicerol, obtendo valores próximos apenas da quantidade de glicerol das cachaças envelhecidas (GARCIA et al., 2015). O glicerol pode influenciar de forma positiva no corpo e na textura da bebida (SANTOS et al., 2013).

As cepas que apresentaram maiores produtividades de etanol para cada ${ }^{\circ}$ Brix consumido foram S.cerevisiae $\operatorname{LNFCA} 11{ }^{\circledR}\left(4,18 \mathrm{~g} / \mathrm{L} /{ }^{\circ} \mathrm{Brix}\right)$ e $S$. cerevisiae LEVFRC29 (3,49 g/L/ ${ }^{\circ}$ Brix) (Tabela 1). O isolado $S$. cerevisiae LEVSIL15 $\left(3,22 \mathrm{~g} / \mathrm{L} /{ }^{\circ} \mathrm{Brix}\right)$ obteve um valor próximo da $S$. cerevisiae LEVFRC29. A cepa $S$. cerevisiae LEVSIL16, em relação às demais, obteve uma produtividade muito baixa $\left(2,08 \mathrm{~g} / \mathrm{L} /{ }^{\circ}\right.$ Brix) (Tabela 1$)$. Todas diferenciam estatisticamente entre si.

Das análises propostas, o propanol, ácido cítrico, ácido succínico e ácido lático não foram detectados nas bebidas.

Pelo fato da polpa de ciriguela possuir elevado teor de sólidos solúveis totais (16 graus brix) próximo ao teor do caldo de cana-de-açúcar, foi dispensável a realização da chaptalização, conservando ainda mais as características sensoriais da fruta na aguardente.

\section{Conclusão}

1. De acordo com os resultados obtidos, podemos concluir que a cepa comercial $S$. cerevisiae LNFCA11 ${ }^{\circledR}$ e $S$. cerevisiae LEVFRC29 obtiveram os melhores resultados, assim como foram capazes de produzir uma aguardente dentro dos padrões do MAPA e obtendo a melhor produtividade em relação às demais cepas avaliadas;

2. O trabalho também apresentou uma metodologia eficiente para produção de aguardente a partir da polpa de ciriguela;

3. Demonstrou que a ciriguela representa um excelente substrato para fermentação alcoólica por $S$. cerevisiae, devido a seu alto teor de sólidos solúveis totais (16 graus brix);

4. Mostrou que a levedura S. cerevisiae LEVFRC29 (isolada pelo grupo de pesquisa (GEFER) a partir da fermentação de silagem) é promissora para a produção de aguardente de ciriguela, visto que seu desempenho foi semelhante àquele da cepa comercial (LNFCA11 $\left.{ }^{\circledR}\right)$.

\section{Agradecimentos}

Agradecemos à UFGD, FUNDECT e CNPq.

\section{Referências}

ALCARDE, A. R.; MONTEIRO, B. M. S.; BELluCO, A. E. S. Composição química de aguardentes de cana-de-açúcar fermentadas por diferentes cepas de levedura Saccharomyces cerevisiae. Química Nova, São Paulo, v. 35, n. 8, p. 1612-1618, 2012. http://dx.doi. org/10.1590/S0100-40422012000800022.

BRASIL. Ministério da Agricultura, Pecuária e Abastecimento - MAPA. Instrução normativa $\mathrm{n}^{\circ} 15$, de 31 de março de 2011. Diário Oficial da República Federativa do Brasil, Brasília, DF, 1 abr. 2011. Disponível em: <https://www.gov.br/agricultura/pt-br/assuntos/ vigilancia-agropecuaria/ivegetal/bebidas-arquivos/in-no-15-de-31de-marco-de-2011.pdf/view>. Acesso em: 11 nov. 2020.

BRASIL. Ministério da Agricultura, Pecuária e Abastecimento - MAPA. Secretaria de Defesa Agropecuária. A cachaça no Brasil: dados de registro de cachaças e aguardentes. Brasília: MAPA/AECE, 2019. Disponível em: <https://www.gov.br/agricultura/pt-br/assuntos/ inspecao/produtos-vegetal/publicacoes/anuario-cachaca.pdf>. Acesso em: 11 nov. 2020 .

CAMPOS, C. R. et al. Features of Saccharomyces cerevisiae as a culture starter for the production of the distilled sugar cane beverage, cachaça in Brazil. Journal of Applied Microbiology, Oxford, v. 108, n. 6, p. 1871-1879, 2010. Disponível em: <https://sfamjournals. onlinelibrary.wiley.com/doi/epdf/10.1111/j.1365-2672.2009.04587. $\mathrm{x}>$. Acesso em: 11 nov. 2020.

GARCIA, A. C. et al. Evaluation of glycerol profiles in sugarcane spirits (cachaças). Journal of the Brazilian Chemical Society, São Paulo, v. 26, n. 1, p. 57-63, 2015. Disponível em: <https:// www.crossref.org/iPage?doi=10.5935\%2F0103-5053.20140213>. Acesso em: 11 nov. 2020.

LIMA, I. C. G. S.; MELEIRO, C. H. A. Desenvolvimento, avaliação físico-química e sensorial de geleia e doce de corte de seriguela (Spondias purpurea L.) visando o crescimento da cadeia produtiva 
do fruto. Digital Library of Journals, Curitiba, v. 30, n. 2, p. 221-232, 2012. Disponível em: <https://revistas.ufpr.br/alimentos/ article/view/30495/20116>. Acesso em: 11 nov. 2020.

LIMA, U. A. Aguardentes. In: AQUARONE, E. et al. (Coord.). Biotecnologia industrial: biotecnologia na produção de alimentos. São Paulo: Edgar Blücher, 2001. cap. 5, v. 4, p. 145-180.

LIMA, U. A.; BASSO, L. C.; AMORIM, H. V. Produção de etanol. In: LIMA, U. A. et al. (Coord.). Biotecnologia industrial: processos fermentativos e enzimáticos. São Paulo: Edgard Blucher, 2001. cap. 1, v. 3, p. 1-43.

MARINHO, A. V.; RODRIGUES, J. P. M.; SIQUEIRA, M. I. D. Avaliação da acidez volátil, teor alcoólico e de cobre em cachaças artesanais. EVS: Estudos Vida e Saúde, Goiânia, v. 36, n. 1-2, p. 75-93, 2009. Disponível em: <http://seer.pucgoias.edu.br/index. php/estudos/article/view/1129>. Acesso em: 11 nov. 2020.

MARTINS, S. T.; MELO, B. Spondias (cajá e outras). 2012. Disponível em: <http://www.fruticultura.iciag.ufu.br/caja.html>. Acesso em: 14 set. 2020.

MENDES FILHO, N. E. et al. Caracterização de aguardentes artesanais de cana-de-açúcar produzidas nas regiões de alpercatas e sertão maranhense. Revista Virtual Química, São Luís, v. 8, n. 5, p. 1421-1432, 2016. http://dx.doi.org/10.21577/1984-6835.20160100.
MENDONÇA, J. G. P. et al. Avaliação dos padrões de identidade e de qualidade de aguardente de frutas. e-xacta, Belo Horizonte, v. 7, n. 2, p. 31-41, 2014. http://dx.doi.org/10.18674/exacta.v7i2.1261.

MUNIZ, C. et al. Bebidas fermentadas a partir de frutos tropicais. Digital Library of Journals, Curitiba, v. 20, n. 2, p. 309-322, 2002. http://dx.doi.org/10.5380/cep.v20i2.1256.

PEREIRA, N. E. et al. Compostos secundários em cachaças produzidas no Estado de Minas Gerais. Ciência e Agrotecnologia, Lavras, v. 27, n. 5, p. 1068-1075, 2003. http://dx.doi.org/10.1590/S1413-70542003000500014.

SANTOS, C. C. A. A. et al. Inoculated fermentation of orange juice (Citrus sinensis L.) for production of a citric fruit spirit. Journal of the Institute of Brewing, London, v. 119, n. 4, p. 280-287, 2013. http://dx.doi.org/10.1002/jib.89.

SILVA, Q. J.; FIGUEIREDO, F. J.; LIMA, V. L. A. G. Características físicas e químicas de cirigueleiras cultivadas na Zona da Mata Norte de Pernambuco. Revista Ceres, Viçosa, v. 63, n. 3, p. 285-290, 2016. http://dx.doi.org/10.1590/0034-737X201663030002.

VIEIRA, C. R. Produção de fermentados a partir de frutas. Belo Horizonte: Fundação Centro Tecnológico de Minas Gerais (CETEC), 2012. Dossiê técnico.

Recebido: 18 maio 202 Aprovado: 11 set. 2021 